\title{
Potencial energético de resíduos sólidos domiciliares do município de Ponta Grossa, Paraná, Brasil
}

\author{
Energy potencial of household solid \\ waste (HSW) in the city of Ponta Grossa, Paraná, Brazil \\ Simone Gomes', Pedro Henrique Weirich Neto², \\ Dimas Agostinho da Silva ${ }^{3}$, Sandra Regina Masetto Antunes ${ }^{4}$, Carlos Hugo Rocha ${ }^{5}$
}

口

\section{RESUMO}

A disposição dos resíduos sólidos domiciliares (RSD) é um desafio na maior parte dos municípios brasileiros, pois é realizada sem tratamento prévio, trazendo inúmeros impactos. A utilização de incineração com recuperação energética possibilita a redução dos impactos. O objetivo deste estudo foi analisar o potencial energético dos RSD do município de Ponta Grossa, Paraná, Brasil. Para isso, foram coletadas amostras no aterro do município para a realização das análises. A caracterização gravimétrica apresentou valores de $0,28 \mathrm{~kg} \mathrm{~kg}^{-1}$ para os recicláveis, $0,44 \mathrm{~kg} \mathrm{~kg}^{-1}$ para a matéria orgânica e 0,28 $\mathrm{kg} \mathrm{kg}^{-1}$ para os rejeitos. Os conteúdos de carbono fixo variaram de 0,06 a $0,09 \mathrm{~kg} \mathrm{~kg}^{-1}$, os de materiais voláteis de 0,75 a $0,81 \mathrm{~kg} \mathrm{~kg}^{-1}$ e os de cinzas de 0,10 a 0,18 kg kg.'. E o poder calorífico superior médio apresentou valor elevado, 19.807 kJ kg', se comparado com o de resíduos de outras cidades do mundo. Palavras-chave: poder calorífico; incineração; recuperação energética.

\begin{abstract}
Household solid waste (HSW) disposition is a challenge for the majority of the Brazilian cities, most held without pretreatment, which causes impacts. Incineration with recovery energy is an alternative to reduce impacts. In this study we analyzed the energy potential of HSW in the city of Ponta Grossa, Paraná, Brazil. Samples were collected at the municipal landfill for the analysis. Waste gravimetric characterization showed value $0.28 \mathrm{~kg} \mathrm{~kg}^{-1}$ for recyclables, $0.44 \mathrm{~kg} \mathrm{~kg}^{-1}$ for organic matter and $0.28 \mathrm{~kg} \mathrm{~kg}^{-1}$ for rejects. The fixed carbon content ranged from 0.06 to $0.09 \mathrm{~kg} \mathrm{~kg}^{-1}$, the volatile materials from 0.75 to $0.81 \mathrm{~kg} \mathrm{~kg}^{-1}$ and ashes from 0.10 to $0.18 \mathrm{~kg} \mathrm{~kg}^{-1}$. The average gross calorific value showed high value, $19,807 \mathrm{~kJ} \mathrm{~kg}^{-1}$, compared to the waste of other cities in the world.
\end{abstract}

Keywords: heat value; incineration; energy recovery.

\section{INTRODUÇÃO}

As fontes de energia disponíveis podem ser classificadas em renováveis e não renováveis. Tal definição é possível mediante sua escala temporal e utilização. As renováveis, no sentido clássico, são aquelas que podem ser repostas pela natureza, apresentando disponibilidade, além de menores impactos ambientais se comparadas às não renováveis. Nesse contexto, os resíduos sólidos domiciliares (RSD) também podem ser considerados fonte de energia renovável, pois são produzidos constantemente e em elevadas quantidades, permanecendo disponíveis por longo tempo na natureza. Os RSD constituem uma das classes do grupo dos resíduos sólidos urbanos que são enviados para os aterros municipais (USEPA, 2010), ou seja, são os resíduos gerados nos domicílios pelos munícipes.
O Brasil registrou um aumento de 35\% na geração de RSD entre 2000 e 2008. E mesmo com o aumento das taxas de reciclagem e compostagem, a quantidade encaminhada para os aterros ainda é elevada, cerca de $90 \%$ da massa gerada (BRASIL, 2011), o que contribui para que os RSD se tornem um dos grandes problemas da atualidade. Isso se dá não apenas pelo esgotamento de algumas áreas devido ao volume ocupado, mas também pela falta de áreas para construção de novos aterros e pelos altos investimentos necessários para tais obras (CHENG \& HU, 2010), além dos custos envolvidos para o monitoramento dos aterros que já foram concluídos, uma vez que continuam produzindo gases e líquidos poluentes e, ainda, outros tipos de contaminação que podem repercutir na saúde pública.

Mestre em Bioenergia pela Universidade Estadual de Ponta Grossa (UEPG). Assessora de Saneamento e Meio Ambiente da Associação dos Municípios do Médio Vale do Itajaí (AMMVI) - Blumenau (SC), Brasil.

${ }^{2}$ Doutor em Engenharia Agrícola pela Universidade Estadual de Campinas (Unicamp). Professor Associado da UEPG - Ponta Grossa (PR), Brasil.

${ }^{3}$ Doutor em Engenharia Florestal pela Universidade Federal do Paraná (UFPR). Professor Associado da UFPR - Curitiba (PR), Brasil.

${ }^{4}$ Doutora em Química pela Universidade Federal de São Carlos (UFSCar). Professora Associada da UEPG - Ponta Grossa (PR), Brasil.

${ }^{5}$ Doutor em Manejo dos Recursos Naturais pela Universidade do Colorado (EUA). Professor Adjunto da UEPG - Ponta Grossa (PR), Brasil.

Endereço para correspondência: Simone Gomes - Rua Alberto Stein, 466 - Velha - 89036-200 - Blumenau (SC), Brasil - E-mail: si.gomes90@gmail.com

Recebido: 01/12/14 - Aceito: 01/11/16 - Reg. ABES: 143432 
Uma possibilidade de redução desses impactos é a combustão controlada dos resíduos - incineração - , permitindo seu tratamento e reduzindo seu volume, seu peso (CHENG et al., 2007; CHENG \& HU, 2010; SINGH et al., 2011), sua periculosidade e suas emissões de poluentes gasosos (CHENG \& HU, 2010; GREGG, 2010; CUCCHIELLA et al., 2012) e líquidos (CHENG \& HU, 2010). A incineração também pode ser vista como um processo de reciclagem, pois apresenta a possibilidade de recuperar energia térmica e elétrica a partir dos RSD (CHENG et al., 2007; CHENG \& HU, 2010; SINGH et al., 2011).

Essa tendência de tratamento é uma alternativa para a demanda de energia - a qual é cada vez maior - , principalmente para os RSD, além de propiciar uma combinação entre benefícios econômicos e ambientais e enfatizar, cada vez mais, que esse tipo de resíduo deve ser visto como um recurso, e não como algo simples que requer eliminação (DENT \& KROL, 1990).

O objetivo desse trabalho foi avaliar o potencial de recuperação de energia a partir da incineração dos RSD do município de Ponta Grossa, Paraná, Brasil.

\section{MATERIAL E MÉTODOS}

O município de Ponta Grossa está localizado na região dos Campos Gerais, centro-sul do estado do Paraná, Brasil. Possui 306.351 habitantes e apresenta índice de desenvolvimento humano (IDH) de 0,763 (IPARDES, 2013). A coleta domiciliar e comercial de Ponta Grossa é dividida em 50 setores do município e os RSD apresentam como destino final o aterro controlado do Botuquara, o qual é utilizado desde 2001. Atualmente, são enviados para o aterro aproximadamente 6.500 Mg de RSD por mês (PGA, 2013).

Para a amostragem, os RSD foram coletados de um caminhão de cada um dos quatro setores estudados. Esses setores englobam de quatro a sete bairros do município e foram escolhidos conforme a sua localização, buscando-se espacializar homogeneamente as amostragens. Os caminhões descarregaram os resíduos no aterro, formando pilhas. De cada pilha foram coletadas quatro amostras de aproximadamente $0,1 \mathrm{~m}^{3} \mathrm{cada}$ (IPT/ CEMPRE, 2000). Esses resíduos coletados foram pesados e depositados sobre uma lona plástica no interior de um quadrado formado por réguas de madeira, com dimensões de 2,00 × 2,00 m, visando a homogeneização.

A seguir foi realizado o processo de quarteamento (IPT/CEMPRE, 2000), no qual se obteve a quantidade de resíduos desejada de cada setor aproximadamente $10 \mathrm{~kg}$-, e suficiente para a realização das análises.

A análise de composição gravimétrica, que consistiu em separar os resíduos manualmente por componente - matéria orgânica, papel, embalagem cartonada, plástico, vidro, metal e rejeito —, foi realizada na Fazenda Escola da Universidade Estadual de Ponta Grossa (UEPG). A análise imediata, que compreende análises de conteúdo de água, carbono fixo, materiais voláteis e cinzas, e a análise de poder calorífico superior (PCS) foram realizadas no Laboratório de Energia de Biomassa Florestal da Universidade Federal do Paraná (UFPR).
Para a realização dessas análises, além dos componentes separados, foram ainda preparadas mais uma amostra de cada setor, denominadas amostras homogeneizadas, as quais são compostas pelas misturas dos componentes nas proporções médias encontradas para cada um, conforme a composição gravimétrica.

A análise imediata foi realizada em duplicata e de acordo com a NBR 8112 (ABNT, 1986), e a de PCS em triplicata e de acordo com a NBR 8633 (ABNT, 1984), utilizando bomba calorimétrica digital IKA, modelo C5000.

\section{RESULTADOS E DISCUSSÃO}

A Tabela 1 apresenta a composição gravimétrica dos quatro setores amostrados.

A maior parte dos RSD enviados para o aterro do município de Ponta Grossa é composta, em média, por $0,42 \mathrm{~kg} \mathrm{~kg}^{-1}$ de matéria orgânica. Embora essa quantidade seja menor que a média nacional $0,51 \mathrm{~kg} \mathrm{~kg}^{-1}$ (BRASIL, 2011) - e também que a disposta em Curitiba $0,48 \mathrm{~kg} \mathrm{~kg}^{-1}$ (MELO et al., 2009) - , esse valor ainda indica um desperdício de matéria-prima e energia pelo seu não reaproveitamento.

Da mesma forma ocorre com os materiais passíveis de reciclagem, sendo destinados $0,31 \mathrm{~kg} \mathrm{~kg}^{-1}$ para o aterro controlado do município. Esses que chegam até os aterros advêm de problemas relacionados à falta de políticas públicas quanto à educação ambiental, visando à segregação desses materiais. O valor de recicláveis encontrado é próximo da média nacional - 0,32 $\mathrm{kg} \mathrm{kg}^{-1}$ - (BRASIL, 2011), o que representa um ponto positivo, pois a geração de resíduos no município está próximo do padrão brasileiro. Por outro lado, também representam desperdício de matéria-prima e energia pelo seu não aproveitamento. Além disso, esses materiais interferem no processo de decomposição dos resíduos e reduzem a área disponível no aterro, pois alguns componentes, como papel, plástico e embalagem cartonada, apresentam baixa densidade.

Tabela 1 - Composição gravimétrica dos resíduos sólidos domiciliares de Ponta Grossa.

\begin{tabular}{c|c|c|c|c|c|c} 
Componentes & $\begin{array}{c}\text { Amos- } \\
\text { tra 1 } \\
\left(\mathrm{kg} \mathrm{kg}^{-1}\right)\end{array}$ & $\begin{array}{c}\text { Amos- } \\
\text { tra 2 } \\
\left(\mathrm{kg} \mathrm{kg}^{-1}\right)\end{array}$ & $\begin{array}{c}\text { Amos- } \\
\text { tra 3 } \\
\left(\mathrm{kg} \mathrm{kg}^{-1}\right)\end{array}$ & $\begin{array}{c}\text { Amos- } \\
\text { tra 4 } \\
\left(\mathrm{kg} \mathrm{kg}^{-1}\right)\end{array}$ & $\begin{array}{c}\text { Média } \\
\left(\mathrm{kg} \mathrm{kg}^{-1}\right)\end{array}$ & $\begin{array}{c}\text { Desvio } \\
\text { Orgânica } \\
\left(\mathrm{kg} \mathrm{kg}^{-1}\right)\end{array}$ \\
\hline Recicláveis & 0,41 & 0,49 & 0,48 & 0,31 & 0,42 & 0,08 \\
\hline Papel & 0,07 & 0,23 & 0,38 & 0,28 & 0,31 & 0,06 \\
\hline $\begin{array}{c}\text { Embalagem } \\
\text { Cartonada }\end{array}$ & 0,02 & 0,02 & 0,02 & 0,02 & 0,02 & 0,00 \\
\hline Plásticos & 0,14 & 0,17 & 0,20 & 0,18 & 0,17 & 0,03 \\
\hline Vidro & 0,04 & 0,00 & 0,04 & 0,05 & 0,03 & 0,02 \\
\hline Metal & 0,06 & 0,02 & 0,03 & 0,01 & 0,03 & 0,02 \\
\hline Rejeito & 0,26 & 0,28 & 0,14 & 0,41 & 0,27 & 0,11 \\
\hline Total & 1,00 & 1,00 & 1,00 & 1,00 & - & - \\
\hline
\end{tabular}


Os outros $0,27 \mathrm{~kg} \mathrm{~kg}^{-1}$ dos resíduos constituem rejeitos que são os únicos materiais que deveriam ser enviados ao aterro, pois não apresentam alternativa viável e/ou conhecida, que não a disposição final (BRASIL, 2011). A quantidade de rejeitos relativa produzida no município de Ponta Grossa é elevada se comparada com a média nacional, que, segundo o Ministério do Meio Ambiente (MMA) (BRASIL, 2011), é de $0,17 \mathrm{~kg} \mathrm{~kg}^{-1}$.

Os valores médios do conteúdo de carbono fixo, materiais voláteis e cinzas das amostras homogeneizadas de RSD dos quatro setores analisados são apresentados na Tabela 2.

O carbono fixo representa a fração que permanece na amostra logo após o aquecimento, ou seja, logo após a liberação dos compostos voláteis (MCKENDRY, 2002). As amostras analisadas apresentaram baixos conteúdos de carbono fixo $\left(0,07 \mathrm{~kg} \mathrm{~kg}^{-1}\right)$ se comparados com o conteúdo presente em madeiras, por exemplo, o qual chega a $0,20 \mathrm{~kg} \mathrm{~kg}^{-1}$ (BARROS et al., 2012). Esse valor baixo é uma vantagem, uma vez que o carbono fixo queima de forma mais lenta (BARROS et al., 2012), sendo necessário maior tempo de residência na câmara de combustão.

As cinzas constituem a parcela não combustível dos RSD, ou seja, o rejeito do processo de combustão (CASTRILLÓN et al., 2013) composto pela fração inorgânica dos resíduos. A amostra 4, assim como a 1 e a 3 , apresentaram conteúdos de cinzas que variaram de 0,17 a $0,18 \mathrm{~kg} \mathrm{~kg}^{-1}$, pois possuem quantidades maiores de material inorgânico $-0,08 \mathrm{~kg} \mathrm{~kg}^{-1}$ em média - se comparadas à amostra 2, que possui $0,02 \mathrm{~kg} \mathrm{~kg}^{-1}$.

As cinzas influenciam no custo do processo de combustão dos RSD e no de transporte, além de contribuir para a corrosão do equipamento (GARCÍA et al., 2013). De acordo com a análise desse resíduo - cinzas - ,

Tabela2-Conteúdo decarbono fixo, materiais voláteise cinzas das amostras homogeneizadas dos resíduos sólidos domiciliares de Ponta Grossa.

\begin{tabular}{l|c|c|c} 
Amostra & $\begin{array}{c}\text { Carbono fixo } \\
\left(\mathrm{kg} \mathrm{kg}^{-1}\right)\end{array}$ & $\begin{array}{c}\text { Materiais } \\
\text { voláteis }\left(\mathrm{kg} \mathrm{kg}^{-1}\right)\end{array}$ & Cinzas $\left(\mathrm{kg} \mathrm{kg}^{-1}\right)$ \\
\hline 1 & 0,07 & 0,76 & 0,17 \\
\hline 3 & 0,09 & 0,81 & 0,10 \\
\hline 4 & 0,06 & 0,76 & 0,17 \\
\hline Média & 0,08 & 0,75 & 0,18 \\
\hline Desvio padrão & 0,07 & 0,77 & 0,15 \\
\hline
\end{tabular}

o mesmo pode apresentar potencial para ser usado como fertilizante ou, ainda, que pode ser empregado como componente em estruturas civis.

O conteúdo de material volátil expressa a facilidade de combustão de uma amostra (MCKENDRY, 2002). A amostra 2 apresentou valor de $0,81 \mathrm{~kg} \mathrm{~kg}^{-1}$. O fator determinante nesse resultado foi o valor de material volátil da matéria orgânica presente nessa amostra, que foi de $0,70 \mathrm{~kg} \mathrm{~kg}^{-1}$ _, como apresentado na Tabela 3 -, valor elevado se comparado com a matéria orgânica das outras amostras, que apresentaram, em média, $0,54 \mathrm{~kg} \mathrm{~kg}^{-1}$ de material volátil.

As diferenças que os materiais orgânicos apresentam na sua composição podem influenciar no seu conteúdo de material volátil, pois alguns elementos, como, por exemplo, o nitrogênio (N) e o enxofre (S), são mais susceptíveis à volatilização do que o cálcio (Ca) (FREITAS \& SANT'ANNA, 2004).

Uma substância é volátil quando apresenta tendência a vaporizar em certas condições de aquecimento (MCKENDRY, 2002). Nas condições avaliadas o componente vidro não é volátil, pois apresentou $0,01 \mathrm{~kg} \mathrm{~kg}^{-1}$ de conteúdo de materiais voláteis. O 1\% de material volátil encontrado se deve à sua coleta ter sido realizada no aterro, no qual os componentes estavam misturados, ocorrendo contaminações do vidro por matéria orgânica ou por outros materiais.

A Tabela 4 apresenta os resultados de conteúdo deágua e do PCS dos quatro setores analisados. O conteúdo de água tem grande interferência no processo de combustão (YANG et al., 2005; LIANG et al., 2008), podendo esta ocorrer mais rápido em amostras com menor conteúdo de água (YANG et al., 2005). Além disso, a umidade dos resíduos contribui para reduções na energia que pode ser recuperada (MCKENDRY, 2002; MENIKPURA \&BASNAYAKE, 2009; GARCÍA et al., 2013). Dessa forma, a secagem prévia dos RSD é um fator importante para melhorar a operação do incinerador e, principalmente, para aumentar a recuperação de energia a partir desses resíduos.

O valor de PCS obtido para a amostra 2 (Tabela 4) pode ser explicado pelo alto conteúdo de materiais voláteis $-0,81 \mathrm{~kg} \mathrm{~kg}^{-1}-$ e pelo baixo conteúdo de cinzas, o que indica conteúdo relativo alto de material interessante para a combustão (LIANG et al., 2008;CUCCHIELLA et al., 2012). Essa amostra também possui quantidade considerável de plástico, o qual também apresenta alto PCS, conforme obtido neste trabalho (Tabelas 5, 6, 7 e 8), bem como relatado por Liu et al. (1996), Oliveira \& Rosa (2003) e Cucchiella et al. (2012).

Tabela 3 - Conteúdo médio de materiais voláteis de cada componente das amostras de resíduos sólidos domiciliares.

\begin{tabular}{l|c|c|c|c|c|c} 
Componentes & $\begin{array}{c}\text { Amostra 1 } \\
\left(\mathrm{kg} \mathrm{kg}^{-1}\right)\end{array}$ & $\begin{array}{c}\text { Amostra 2 } \\
\left(\mathrm{kg} \mathrm{kg}^{-1}\right)\end{array}$ & $\begin{array}{c}\text { Amostra 3 } \\
\left(\mathrm{kg} \mathrm{kg}^{-1}\right)\end{array}$ & $\begin{array}{c}\text { Amostra 4 } \\
\left(\mathrm{kg} \mathrm{kg}^{-1}\right)\end{array}$ & $\begin{array}{c}\text { Média } \\
\left(\mathrm{kg} \mathrm{kg}^{-1}\right)\end{array}$ & $\begin{array}{c}\text { Desvio padrão } \\
\left(\mathrm{kg} \mathrm{kg}^{-1}\right)\end{array}$ \\
\hline Matéria orgânica & 0,56 & 0,70 & 0,53 & 0,53 & 0,08 \\
\hline Papel & 0,77 & 0,77 & 0,77 & 0,77 & 0,77 \\
\hline Embalagem cartonada & 0,79 & 0,79 & 0,79 & 0,79 & 0,79 & 0,00 \\
\hline Plástico & 0,91 & 0,91 & 0,91 & 0,91 & 0,91 & 0,00 \\
\hline Vidro & 0,01 & 0,01 & 0,01 & 0,01 & 0,01 \\
\hline Metal & 0,01 & 0,01 & 0,01 & 0,01 & 0,01 & 0,00 \\
\hline
\end{tabular}


A amostra 3 apresentou valor médio de PCS próximo do valor da amostra 2, pois possui grande quantidade de plástico e papel na sua composição. No entanto, a amostra 3, assim como a 1 e a 4, apresentaram maiores conteúdos de cinzas devido à maior quantidade de material inorgânico presente, o que contribuiu para a redução de seus PCS (Tabela 4).

Os PCS de todas as amostras analisadas estão próximos dos encontrados por Soares (2011) para municípios do Rio de Janeiro, que obteve, em média, o valor de $18.134 \mathrm{~kJ} \mathrm{~kg}^{-1}$. Os RSD da cidade de Kuala Lumpur apresentam valor bem abaixo dos encontrados —, $9.127 \mathrm{~kJ} \mathrm{~kg}^{-1}$ - , o que se deve ao alto conteúdo de água presente $-0,55 \mathrm{~kg} \mathrm{~kg}^{-1}-\mathrm{e}$ ao baixo conteúdo de materiais voláteis $-0,31 \mathrm{~kg} \mathrm{~kg}^{-1}-$ (KATHIRVALE et al., 2003), bem como, provavelmente, devido às diferenças nas composições dos resíduos, nos tipos de materiais, na cultura e, ainda, temporalmente.

Tabela 4 - Conteúdo de água, poder calorífico superior , poder calorífico superior médio, desvio padrão e coeficiente de variação das amostras homogeneizadas de resíduos sólidos domiciliares.

\begin{tabular}{|c|c|c|c|c|c|}
\hline Amostra & $\begin{array}{l}\text { Conteúdo } \\
\text { de água } \\
\left(\mathrm{kg} \mathrm{kg}^{-1}\right)\end{array}$ & $\begin{array}{c}\text { PCS } \\
\left(\mathrm{kJ} \mathrm{kg}^{-1}\right)\end{array}$ & $\begin{array}{c}\text { PCS } \\
\text { médio } \\
\left(\mathrm{kJ} \mathrm{kg}^{-1}\right)\end{array}$ & $\begin{array}{l}\text { Desvio } \\
\text { padrão } \\
\left(\mathrm{kJ} \mathrm{kg}^{-1}\right)\end{array}$ & $\begin{array}{l}\text { Coeficiente } \\
\text { de variação } \\
\text { (\%) }\end{array}$ \\
\hline \multirow{3}{*}{1} & \multirow{3}{*}{0,36} & 22.320 & \multirow{3}{*}{20.317} & \multirow{3}{*}{1.776} & \multirow{3}{*}{8,74} \\
\hline & & 18.933 & & & \\
\hline & & 19.699 & & & \\
\hline \multirow{3}{*}{2} & \multirow{3}{*}{0,44} & 20.076 & \multirow{3}{*}{21.590} & \multirow{3}{*}{3.129} & \multirow{3}{*}{14,49} \\
\hline & & 25.188 & & & \\
\hline & & 19.506 & & & \\
\hline \multirow{3}{*}{3} & \multirow{3}{*}{0,40} & 21.223 & \multirow{3}{*}{21.224} & \multirow{3}{*}{2.502} & \multirow{3}{*}{11,79} \\
\hline & & 18.723 & & & \\
\hline & & 23.727 & & & \\
\hline \multirow{3}{*}{4} & \multirow{3}{*}{0,33} & 19.217 & \multirow{3}{*}{19.735} & \multirow{3}{*}{489} & \multirow{3}{*}{2,48} \\
\hline & & 19.799 & & & \\
\hline & & 20.189 & & & \\
\hline
\end{tabular}

PCS: poder calorífico superior.

Tabela 5 - Cálculo do poder calorífico superior médio por componente da amostra 1, considerando a composição gravimétrica dessa amostra.

\begin{tabular}{l|c|c|c|c} 
Componente & $\begin{array}{c}\text { PCS } \\
\left(\mathrm{kJ} \mathrm{kg}^{-1}\right)\end{array}$ & $\begin{array}{c}\text { Quantidade } \\
\text { de RSU } \\
(\mathrm{kg})\end{array}$ & $\begin{array}{c}\text { Composição } \\
(\%)\end{array}$ & $\begin{array}{c}\text { PCS do } \\
\text { componente } \\
\left(\mathrm{kJ} \mathrm{kg}^{-1}\right)\end{array}$ \\
$\begin{array}{l}\text { Matéria } \\
\text { orgânica }\end{array}$ & 15.995 & 4,20 & 41,00 & 6.558 \\
\hline Papel & 15.740 & 0,73 & 7,00 & 1.102 \\
\hline $\begin{array}{l}\text { Embalagem } \\
\text { cartonada }\end{array}$ & 22.157 & 0,16 & 2,00 & 443 \\
\hline Plástico & 35.937 & 1,43 & 14,00 & 5.031 \\
\hline Vidro & 0,00 & 0,44 & 4,00 & 0,00 \\
\hline Metal & 0,00 & 0,56 & 6,00 & 0,00 \\
\hline Rejeito & 18.461 & 2,66 & 26,00 & 4.800 \\
\hline Total & & 10,18 & 100,00 & 17.934 \\
\hline
\end{tabular}

PCS: poder calorífico superior; RSD: resíduos sólidos domiciliares.
Tabela 6 - Cálculo do poder calorífico superior médio por componente da amostra 2, considerando a composição gravimétrica desta amostra.

\begin{tabular}{l|c|c|c|c} 
Componente & $\begin{array}{c}\text { PCS } \\
\left(\mathrm{kJ} \mathrm{kg}^{-1}\right)\end{array}$ & $\begin{array}{c}\text { Quantidade } \\
\text { de RSU } \\
(\mathrm{kg})\end{array}$ & $\begin{array}{c}\text { Composição } \\
(\%)\end{array}$ & $\begin{array}{c}\text { PCS do } \\
\text { componente } \\
\left(\mathrm{KJ} \mathrm{kg}^{-1}\right)\end{array}$ \\
$\begin{array}{l}\text { Matéria } \\
\text { orgânica }\end{array}$ & 17.331 & 3,99 & 49,00 & 8.492 \\
\hline Papel & 15.740 & 0,17 & 2,00 & 315 \\
\hline $\begin{array}{l}\text { Embalagem } \\
\text { cartonada }\end{array}$ & 22.157 & 0,12 & 2,00 & 443 \\
\hline Plástico & 35.937 & 1,34 & 17,00 & 6.109 \\
\hline Vidro & 0,00 & 0,03 & 0,00 & 0,00 \\
\hline Metal & 0,00 & 0,15 & 2,00 & 0,00 \\
\hline Rejeito & 18.461 & 2,29 & 28,00 & 5.169 \\
\hline Total & & 8,09 & 100,00 & 20.528 \\
\hline
\end{tabular}

PCS: poder calorífico superior; RSD: resíduos sólidos domiciliares.

Tabela 7 - Cálculo do poder calorífico superior médio por componente da amostra 3, considerando a composição gravimétrica dessa amostra.

\begin{tabular}{|c|c|c|c|c|}
\hline Componente & $\begin{array}{c}\text { PCS } \\
\left(\mathrm{kJ} \mathrm{kg}^{-1}\right)\end{array}$ & $\begin{array}{l}\text { Quantidade } \\
\text { de RSU } \\
\text { (kg) }\end{array}$ & $\begin{array}{c}\text { Composição } \\
\text { (\%) }\end{array}$ & $\begin{array}{l}\text { PCS do } \\
\text { componente } \\
\left(\mathrm{kJ} \mathrm{kg}^{-1}\right)\end{array}$ \\
\hline $\begin{array}{l}\text { Matéria } \\
\text { orgânica }\end{array}$ & 14.026 & 3,71 & 48,00 & 6.732 \\
\hline Papel & 15.740 & 0,69 & 9,00 & 1.417 \\
\hline $\begin{array}{l}\text { Embalagem } \\
\text { cartonada }\end{array}$ & 22.157 & 0,16 & 2,00 & 443 \\
\hline Plástico & 35.937 & 1,55 & 20,00 & 7.187 \\
\hline Vidro & 0,00 & 0,34 & 4,00 & 0,00 \\
\hline Metal & 0,00 & 0,22 & 3,00 & 0,00 \\
\hline Rejeito & 18.461 & 1,09 & 14,00 & 2.585 \\
\hline Total & & 7,76 & 100,00 & 18.364 \\
\hline
\end{tabular}

PCS: poder calorífico superior; RSD: resíduos sólidos domiciliares.

Tabela 8 - Cálculo do poder calorífico superior médio por componente da amostra 4, considerando a composição gravimétrica dessa amostra.

\begin{tabular}{l|c|c|c|c} 
Componente & $\begin{array}{c}\mathrm{PCS} \\
\left(\mathrm{KJ} \mathrm{kg}^{-1}\right)\end{array}$ & $\begin{array}{c}\text { Quantidade } \\
\text { de RSU } \\
(\mathrm{kg})\end{array}$ & $\begin{array}{c}\text { Composição } \\
(\%)\end{array}$ & $\begin{array}{c}\text { PCS do } \\
\text { (omponente } \\
\left(\mathrm{kJ} \mathrm{kg}^{-1}\right)\end{array}$ \\
$\begin{array}{l}\text { Matéria } \\
\text { Orgânica }\end{array}$ & 12.806 & 3,37 & 31,00 & 3.970 \\
\hline Papel & 15.740 & 0,25 & 2,00 & 315 \\
\hline $\begin{array}{l}\text { Embalagem } \\
\text { cartonada }\end{array}$ & 22.157 & 0,22 & 2,00 & 443 \\
\hline Plástico & 35.937 & 1,94 & 18,00 & 6.469 \\
\hline Vidro & 0,00 & 0,53 & 5,00 & 0,00 \\
\hline Metal & 0,00 & 0,07 & 1,00 & 0,00 \\
\hline Rejeito & 18.461 & 4,44 & 41,00 & 7.569 \\
\hline Total & & 10,82 & 100,00 & 18.766 \\
\hline PCS: poder calo
\end{tabular}

PCS: poder calorífico superior; RSD: resíduos sólidos domiciliares. 
As Tabelas 5, 6, 7 e 8 mostram os resultados de PCS obtidos para cada componente das amostras. Não foi possível realizar a análise de PCS do componente embalagem cartonada, pois poderia ocasionar danos no equipamento utilizado. Portanto, foi adotado o valor teórico de 22.156,55 kJ kg-1 (CEMPRE, 2000), o qual se aproxima do valor analisado por Montejo et al. (2011), que foi de $23.557,10 \mathrm{~kJ} \mathrm{~kg}^{-1}$.

A matéria orgânica apresentou PCS de $14.566 \mathrm{~kJ} \mathrm{~kg}^{-1}$, em média, próximo ao componente papel - $15.512 \mathrm{~kJ} \mathrm{~kg}^{-1}$. Por sua vez, o valor de PCS do componente plástico foi, em média, $37.018 \mathrm{~kJ} \mathrm{~kg}^{-1}$. Os valores encontrados estão próximos aos referenciados (Tabela 9).

$\mathrm{Na}$ Figura 1 estão apresentadas as médias entre o PCS das amostras homogeneizadas e os calculados por componentes. As homogeneizadas

Tabela 9 - Comparação do poder calorífico superior ( $\left.\mathrm{kJ} \mathrm{kg}^{-1}\right)$ encontrado para os componentes matéria orgânica, papel e plástico com bibliografia correlata.

\begin{tabular}{l|c|c|c|c} 
Componentes & Encontrado & $\begin{array}{c}\text { Komilis et al. } \\
\text { (2012) }\end{array}$ & $\begin{array}{c}\text { Montejo } \\
\text { et al. (2011) }\end{array}$ & $\begin{array}{c}\text { Menikpura } \\
\text { \& Basnayake } \\
\text { (2009) }\end{array}$ \\
$\begin{array}{l}\text { Matéria } \\
\text { Orgânica }\end{array}$ & 14.566 & 16.316 & 14.905 & - \\
\hline Papel & 15.512 & 14.268 & 14.740 & 15.680 \\
\hline Plástico & 37.018 & 39.350 & 37.785 & 39.150 \\
\hline
\end{tabular}

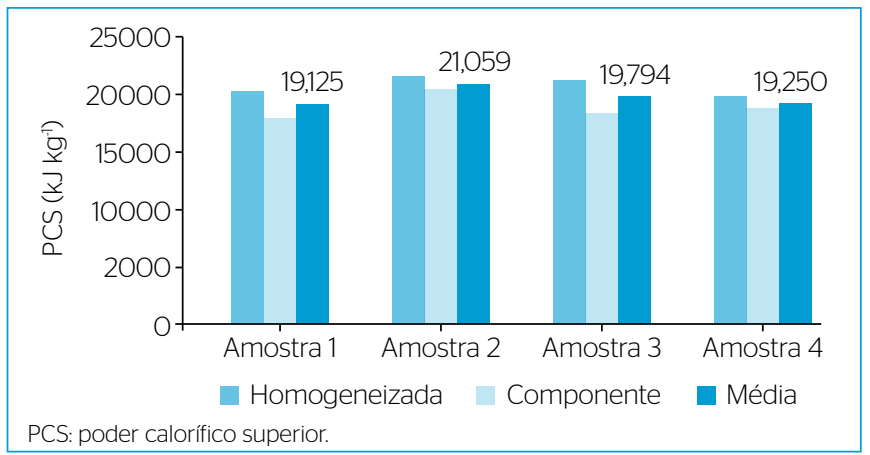

Figura 1 - Comparação entre as médias de poder calorífico superior obtidas nas amostras homogeneizadas e o poder calorífico superior obtido por componente. apresentaram maior valor de PCS em todas as amostras se comparados com os calculados por componente. Mesmo que as amostras homogeneizadas tenham sido preparadas nas proporções certas encontradas na composição gravimétrica, sua introdução na bomba calorimétrica para análise é subjetiva, uma vez que a quantidade colocada para ser analisada é muito pequena. No entanto, resultados semelhantes a esse supramencionado - valor de PCS de amostras homogeneizadas maior que o calculado por componente - foram encontrados por Soares (2011).

Os RSD de Ponta Grossa apresentam um alto valor de PCS quando comparado com os de outros países, como, por exemplo, os da Malásia, que se situa entre 6.280 e $10.880 \mathrm{~kJ} \mathrm{~kg}^{-1}$ (KATHIRVALE et al., 2003), o da Índia, que é de $10.008 \mathrm{~kJ} \mathrm{~kg}^{-1}$ (KUMAR \& GOEL, 2009), o da China, $5.000 \mathrm{~kJ} \mathrm{~kg}^{-1}$ (CHENG \& HU, 2010) e o da região Norte da Espanha, $11.113 \mathrm{~kJ} \mathrm{~kg}^{-1}$ (CASTRILLÓN et al., 2013).Vale ressaltar que alguns desses países - China e Espanha - já utilizam a energia proveniente dos resíduos sólidos urbanos (RSU). Dessa forma, seria interessante a recuperação dessa energia no município de Ponta Grossa, principalmente considerando que a Política Nacional de Resíduos Sólidos (PNRS) (BRASIL, 2010) prevê a utilização dessas tecnologias na gestão dos resíduos sólidos.

\section{CONCLUSÃO}

- A maior parte dos RSD do município de Ponta Grossa é composta por matéria orgânica - aproximadamente $40 \%$ —, cerca de $30 \%$ são materiais passíveis de reciclagem e os outros $30 \%$ constituem os rejeitos;

- As amostras de RSD apresentaram baixos valores de carbono fixo0,06 a $0,09 \mathrm{~kg} \mathrm{~kg}^{-1}$. O conteúdo de materiais voláteis variou de 0,75 a $0,81 \mathrm{~kg} \mathrm{~kg}^{-1}$ e o de cinzas de 0,10 a $0,18 \mathrm{~kg} \mathrm{~kg}^{-1}$;

- O conteúdo de água presente nos RSD variou de 0,33 a $0,44 \mathrm{~kg} \mathrm{~kg}^{-1}$;

- O PCS variou de 19.125 a $21.059 \mathrm{~kJ} \mathrm{~kg}^{-1}$, com PCS médio de $19.807 \mathrm{~kJ} \mathrm{~kg}^{-1}$.

\section{REFERÊNCIAS}

ABNT - Associação Brasileira de Normas Técnicas. (1984) NBR 8633: carvão vegetal, determinação do poder calorífico - método de ensaio. Rio de Janeiro.

ABNT - Associação Brasileira de Normas Técnicas. (1986) NBR 8112: carvão vegetal - análise imediata. Rio de Janeiro.

BARROS, S.V.S.; NASCIMENTO, C.C.; AZEVEDO, C.P. (2012) Caracterização tecnológica da madeira de três espécies florestais cultivadas no Amazonas: alternativa para produção de lenha. Floresta, v. 42, n. 4, p. 725- 732.
BRASIL. (2010) Presidência da República. Lei n. 12.305 , de 2 de agosto de 2010. Institui a Política Nacional de Resíduos Sólidos; altera a Lei n.o 9.605, de 12 de fevereiro de 1998; e dá outras providências. Diário Oficial da União, Brasília.

BRASIL. (2011) MMA - Ministério do Meio Ambiente. Plano Nacional de Resíduos Sólidos: versão preliminar para consulta pública. Brasília.

CASTRILLÓN, L.; FERNÁNDEZ-NAVA, Y.; GONZÁLEZ, A.; MARAÑÓN, E. (2013) A case study of the characteristics 
of municipal solid waste in Asturias (Spain): influence of season and source. Waste Management \& Research, v. 31, n. 4, p. 428-431.

CEMPRE - Compromisso Empresarial para Reciclagem. (2000) Embalagem cartonada: Ionga vida - o mercado para reciclagem. Disponível em: <http://www.ecologia.dbi.ufla.br/site\%2Oecoaplicada/ reciclagem/Embalagem\%2OCartonada.doc>. Acesso em: 17 dez. 2013.

CHENG, H. \& HU, Y. (2010) Municipal solid waste (MSW) as a renewable source of energy: current and future practices in China. Bioresource Technology, v. 101, p. 3816-3824.

CHENG, H.; ZHANG, Y.; MENG, A.; LI, Q. (2OO7) Municipal solid waste fueled power generation in China: a case study of waste-to-energy in Changchun city. Environmental Science \& Technology, v. 41, n. 21.

CUCCHIELLA, F.; D’ADAMO, I.; GASTALDI, M. (2012) Municipal waste management and energy recovery in an Italian region. Waste Management \& Research, v. 30, n. 12, p. 1290-1298.

DENT, C.G. \& KROL, A.A. (1990) Municipal solid waste conversion to energy. Biomass, v. 22, p. 307-327.

FREITAS, L.C. \& SANT'ANNA, G.L. (2004) Efeitos do fogo nos ecossistemas florestais. Revista da Madeira, n. 79.

GARCÍA, R.; PIZARRO, C.; LAVÍN, A.G.; BUENO, J.L. (2013) Biomass proximate analysis using thermogravimetry. Bioresource Technology, v. 139C, p. 1-4.

GREGG, J.S. (2010) National and regional generation of municipal residue biomass and the future potential for waste-to-energy implementation. Biomassand Bioenergy, v. 34, p. 379-388.

IPARDES - Instituto Paranaense de Desenvolvimento Econômico e Social. (2013) Caderno estatístico do município de Ponta Grossa. Curitiba: IPARDES. Disponível em: <http://www.ipardes.gov.br/ cadernos/Montapdf.php?Municipio=84000>. Acesso em: fev. 2014.

IPT/CEMPRE - Instituto de Pesquisas Tecnológicas do Estado de São Paulo/Compromisso Empresarial para Reciclagem.(2000) Lixo municipal: Manual de Gerenciamento Integrado. $2^{a}$ ed. São Paulo. 370 p.

KATHIRVALE, S.; YUNUS, M.N.M.; SOPIAN, K.SAMSUDDIN, A.H. (2004) Energy potential from municipal solid waste in Malaysia. Renewable Energy, v. 29, p. 559-567.

KOMILIS, D.; EVANGELOU, A.; GIANNAKIS, G.; LYMPERIS, C. (2O12) Revisiting the elemental composition and the calorific value of the organic fraction of municipal solid wastes. Waste Management, v. 32, p. 372-381.

KUMAR, K.N. \& GOEL, S. (2009) Characterization of Municipal Solid Waste (MSW) and a proposed management plan for
Kharagpur,West Bengal, India. Resources, Conservation and Recycling, v. 53, p. 166-174.

LIANG, L.; SUN, R.; FEI, J.; WU, S.; LIU, X.; DAI, K.; YAO, N. (2008) Experimental study on effects of moisture content on combustion characteristics of simulated municipal solid wastes in a fixed bed Bioresource Technology, v. 99, p. 7238-7246.

LIU, J-I.; PAODE, R.D.; HOLSEN, T.M. (1996) Modeling the energy content of municipal solid waste using multiple regression analysis. Journal of the Air \& Waste Management Association, v. 46, n. 7. p. 650-656.

MCKENDRY, P. (2002) Energy production from biomass (part 1): overview of biomass. Bioresource Technology, v. 83, p. 37-46.

MELO, L.A.; SAUTTER, K.D.; JANISSEK, P.R. (2009) Estudo de cenários para o gerenciamento dos resíduos sólidos urbanos de Curitiba. Engenharia Sanitária e Ambiental, v. 14, n. 4.

MENIKPURA, S.N.M. \& BASNAYAKE, B.F.A. (2009) New applications of "Hess Law" and comparisons with models for determining calorific values of municipal solid wastes in the Sri Lankan context. Renewable Energy, v. 34, p. 1587-1594.

MONTEJO, C.; COSTA, C.; RAMOS, P.; MÁRQUEZ, M.C. (2011) Analysis and comparison of municipal solid waste and reject fraction as fuels for incineration plants. Applied Thermal Engineering, v. 31, p. $2135-2140$.

OLIVEIRA, L.B. \& ROSA, L.P. (2003) Brazilian waste potential: energy, environmental, social and economic benefits. Energy Policy, v. 31, p.1481-1491.

PGA - Ponta Grossa Ambiental. (2013) Informações sobre a quantidade de resíduos coletados. Ponta Grossa.

SINGH, R.P.; TYAGI, V.V.; ALLEN, T.; IBRAHIM, M.H.; KOTHARI, R. (2O11) An overview for exploring the possibilities of energy generation from municipal solid waste (MSW) in Indian scenario. Renewable and Sustainable Energy Reviews, v. 15, p. 4797-4808.

SOARES, E.L.S.F. (2011) Estudo da caracterização gravimétrica e poder calorífico dos resíduos sólidos urbanos. 133p. Dissertação (Mestrado) - Universidade Federal do Rio de Janeiro, Rio de Janeiro.

USEPA - United Environmental Protection Agency. (2010) Municipal solid waste in the United States: 2009 facts and figures. Estados Unidos.

YANG, Y.B.; RYU, C.; KHOR, A.; YATES, N.E.; SHARIFI, V.N.; SWITHENBANK, J. (2005) Effect of fuel properties on biomass combustion. Part II. Modelling approach-identification of the controlling factors. Fuel, v. 84, p. 2116-2130. 